\title{
AVALIAÇÃO DA LEISHMANIOSE VISCERAL CANINA EM POXORÉO, ESTADO DO MATO GROSSO, BRAZIL
}

\author{
MÁRCIA ÁVILA A. DE AZEVEDO'; ; ANA KARINA K. DIAS²; HENRIQUE B. DE PAULA; \\ SILVIA HELENA V. PERRI ${ }^{3}$; CÁRIS M. NUNES ${ }^{3}$
}

\begin{abstract}
AZEVEDO, M.A. DE ; DIAS, A.K.K.; PAULA, H.B. DE.; PERRI, S.H.V.; NUNES, C.M. [Canine visceral leishmaniasis evaluation in Poxoréo, Mato Grosso State, Brazil.] Avaliação da leishmaniose visceral canina em Poxoréo, Estado do Mato Grosso, Brasil. Revista Brasileira de Parasitologia Veterinária, v. 17, n. 3, p. 123-127, 2008. Curso de Medicina Veterinária, Departamento de Apoio, Produção e Saúde Animal/UNESP, Rua Clóvis Pestana, 793, Jardim D. Amélia, Araçatuba, SP 16050-680, Brasil. E-mail: caris@fmva.unesp.br

Dogs play an important role as reservoir in the domestic cycle of visceral leishmaniasis, a serious public health problem. An epidemiological survey in 1,112 dogs was conducted at the Municipality of Poxoréo State of Mato Grosso, Brazil, using indirect immunofluorescence antibody test where the prevalence was $7.8 \%$. Significant association was found between prevalence of canine visceral leishmaniasis and age of the dogs. Clinical signs and presence of other animals in the backyard, like chicken being more likely associated with seropositivity. Gender, garbage collection in the residence and family financial income were not associated with visceral leishmaniasis prevalence. Analysis of the results suggests that dogs aging more than 7 years and presence of another animal species co-inhabiting with the dogs may be risk factors for canine visceral leishmaniasis.
\end{abstract}

KEY WORDS: Risk factors, epidemiology, prevalence, dogs.

\section{RESUMO}

O cão doméstico desempenha importante papel como reservatório na transmissão da leishmaniose visceral ao homem, zoonose de grande importância em saúde pública. Realizou-se avaliação epidemiológica da leishmaniose visceral em 1.112 cães domiciliados no município de Poxoréo, estado do Mato Grosso e observou-se prevalência de 7,8\%. Observou-se ainda associação estatisticamente significativa entre a prevalência de leishmaniose visceral canina e as variáveis faixa etária, presença de sinais clínicos e presença de outra espécie animal cohabitando com os cães avaliados, tendo sido as galinhas mais freqüentemente observadas entre os animais soropositivos. $\mathrm{O}$ sexo, a coleta de lixo domiciliar bem como a renda familiar não apresentaram associação estatisticamente significativa com a prevalência da leishmaniose visceral canina. A análise dos re-

\footnotetext{
${ }^{1}$ Pós-Graduação, Faculdade de Medicina Veterinária e Zootécnica, Departamento de Higiene Veterinária e Saúde Pública, Universidade Estadual Paulista “Júlio de Mesquita Filho”, Unesp-Botucatu, Distrito de Rubião Júnior, s/n, Botucatu, SP 18618-000, Brasil.

${ }^{2}$ Pós-Graduação, Instituto de Biociências, Unesp-Botucatu, SP.

${ }^{3}$ Curso de Medicina Veterinária, Departamento de Apoio, Produção e Saúde Animal, Unesp-Araçatuba, Rua Clóvis Pestana, 793, Jardim D. Amélia, Araçatuba, SP 16050-680, Brasil. E-mail: caris@fmva.unesp.br
}

sultados sugere que cães com idade superior a sete anos e a , presença de outra espécie animal co-habitando com os cães podem ser fatores de risco para a leishmaniose visceral canina.

PALAVRAS-CHAVE: Fatores de risco, epidemiologia, prevalência, cães.

\section{INTRODUÇÃO}

As leishmanioses são causadas por protozoários do gênero Leishmania, parasita que infecta grande número de mamíferos, inclusive o ser humano, e cuja transmissão depende da picada de flebotomíneos. A leishmaniose visceral (LV) é a forma mais severa da doença e invariavelmente leva ao óbito, se não tratada (GRAMICCIA; GRADONI, 2005). O baixo padrão de vida da população, a maior densidade dos flebotomíneos e cães infectados são fatores predisponentes para implantação desta zoonose (ALENCAR, 1983; BADARÓ et al., 1986; MONTEIRO et al., 1994). A leishmaniose visceral humana nem sempre obedece a uma distribuição espacial paralela a do calazar canino, porém as infecções caninas são mais freqüentes que as humanas e normalmente as precedem (PARANHOS-SILVA et al., 1996).

No Brasil, a LV ocorre endemicamente em várias regiões e tem apresentado expansão e urbanização, porém há pouco 
conhecimento sobre a epidemiologia da doença neste novo cenário (GONTIJO; MELO, 2004).

A prevalência da leishmaniose visceral canina (LVC) tem sido objeto de estudo em diferentes regiões do Brasil (PARANHOS-SILVA et al., 1996; MADEIRA et al., 2000; CAMARGO-NEVES et al., 2001; FRANÇA-SILVA et al., 2003; CABRERA et al., 2003; CORTADA et al., 2004; GUIMARÃES et al., 2005) com resultados que variam de acordo com as características da população, bem como com a metodologia empregada na avaliação.

Fatores de risco como faixa etária, raça, tamanho e tipo do pelame, estado geral, sintomatologia clínica e condições do peridomicílio onde vivem os cães (FEITOSA et al., 2000; ALEXANDER et al., 2002; CABRERA et al., 2003; MOREIRA Jr et al., 2003, 2004; FRANÇA-SILVA et al., 2003; GUIMARÃES et al., 2005; COSTA et al., 2005) têm sido avaliados na ocorrência de LVC.

A região de Poxoréo, no estado do Mato Grosso, tem registrado casos humanos de leishmaniose visceral e as medidas aplicadas no município para o controle desta enfermidade têm sido a identificação e tratamento de casos humanos, a identificação e eutanásia de cães soropositivos e o controle vetorial. O conhecimento de parâmetros epidemiológicos, além da quantificação da doença, pode auxiliar quando da implementação de medidas de controle. Assim sendo, objetivou-se avaliar a prevalência da LVC em cães domiciliados no município de Poxoréo e associar esta ocorrência a dados como faixa etária, sexo, presença de animais no peridomicílio, coleta de lixo e renda familiar.

\section{MATERIAL E MÉTODOS}

\section{Área de Estudo}

O Município de Poxoréo está localizado no sudeste Mato-

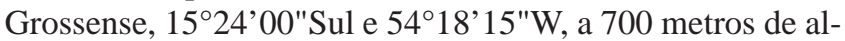
titude. O clima é do tipo tropical quente e subúmido, com temperatura média de $22^{\circ} \mathrm{C}$, estação seca de maio a agosto e estação chuvosa de dezembro a fevereiro. A densidade demográfica é de $2,84 \mathrm{hab} / \mathrm{km}^{2}$, a população foi estimada em 20.030 habitantes em 2000, distribuídos em aproximadamente 5.500 domicílios (BRASIL, 2000). A região possui atividades comerciais e rurais voltadas para o garimpo e a pecuária bovina em sistema extensivo, as quais têm modificado bastante a paisagem natural. O município já teve registro de casos humanos e caninos de leishmaniose visceral.

\section{Amostragem}

O tamanho mínimo da amostra foi calculado em 864 cães, ao nível de confiança de $95 \%$, precisão absoluta de $2 \%$ e prevalência esperada de 10\% (LWANGA; LEMESHOW, 1991). A amostra foi estratificada utilizando-se a divisão de setores já existente na área urbana, segundo a área de trabalho da Fundação Nacional de Saúde (FUNASA). Considerando-se que não havia dados prévios referentes ao tamanho e distribuição da população canina no município de Poxoréo, adotou-se a relação de um cão para cada 10 habitantes (CIFUENTES, 1988) para avaliação da distribuição dos cães nos setores. A seleção da amostra foi feita de forma aleatória, proporcionalmente ao número médio de cães estimado por setor.

\section{Coleta de material}

No período de setembro a novembro de 2002 foram coletadas informações através de um questionário aplicado aos proprietários além de amostras de sangue, através da punção da veia cefálica, de 1.112 cães domiciliados, com idade categorizada em cinco faixas etárias (de zero a um ano; de um a três anos; de três a cinco anos; de cinco a sete anos e maior que sete anos) e raças variadas, após consentimento esclarecido dos proprietários. O número de amostras coletadas foi maior que o calculado, pois, em alguns setores, o número de animais era diferente do estimado. No ato da coleta do material foram realizados exame clínico sumário e classificação do cão em assintomático e sintomático, segundo a ausência ou a presença, respectivamente, de um ou mais sinais clínicos sugestivos de LVC (alopecia, lesões cutâneas, onicogrifose, infartamento ganglionar e ceratoconjuntivite). Tal avaliação foi realizada pela médica veterinária que coletou as amostras. Neste momento realizou-se também a inspeção visual dos locais onde viviam os cães, bem como o registro da presença de outras espécies animais (galinhas, suínos e eqüinos) co-habitando com os cães. A presença de coleta de lixo foi registrada segundo informação dos residentes.

A presente pesquisa teve a aprovação da Câmara de Ética em Experimentação Animal da Faculdade de Medicina Veterinária e Zootecnia da UNESP/Botucatu (05/2002- CEEA).

\section{Análise laboratorial}

A presença de anticorpos anti-Leishmania foi avaliada através da reação imunofluorescência indireta (RIFI), realizada pelo Laboratório Central do Estado do Mato Grosso (LACEN), utilizando-se o "kit” IFI-Leishmaniose canina (Biomanguinhos, Fiocruz) segundo Camargo e Rebonato (1969). A diluição discriminante do soro foi de 1:40.

\section{Análise estatística}

A avaliação de associação entre as variáveis (sexo, faixa etária, presença de sinais clínicos, condições do peridomicílio, coleta de lixo domiciliar e renda familiar) estudadas e o resultado da avaliação sorológica foi realizada pelo teste do Qui quadrado $\left(\chi^{2}\right)$ ou teste exato de Fisher, por meio do programa SAS $^{\circledR}$ (SAS, 1999), com nível de significância de 5\%.

\section{RESULTADOS}

A prevalência de anticorpos anti-Leishmania foi 7,8\% (87/ 1.112) na amostra analisada, com presença de cães positivos em todos os Setores do município de Poxoréo

Dentre os animais avaliados, 62,1\% (691/1.112) eram do sexo masculino, 35,8\% (398/1.112) do sexo feminino e em vinte e três registros $(2,1 \%)$ as informações sobre sexo não foram anotadas. A ocorrência de anticorpos anti-Leishmania 
nos machos foi de 8,2\% (57/691) enquanto que nas fêmeas foi de 7,5\% (30/398), diferença estatisticamente não significativa $(\mathrm{p}=0,6768)$.

Com relação à distribuição etária, a população era composta principalmente por animais entre 1 e 3 anos de idade, seguida de animais de até um ano de idade, caracterizando uma população jovem. Em 27,0\% dos registros (303/1.112) as informações sobre a idade dos animais não foram anotadas. A ocorrência de anticorpos anti-Leishmania foi observada em todas as faixas etárias. Os animais com mais de sete anos de idade apresentaram maior percentual de positividade, não diferindo da faixa de animais entre cinco e sete anos, mas com diferença estatisticamente significativa das demais (Tabela 1).

A avaliação clínica não foi registrada em 7,6\% dos cães (84/1.112). Dos animais cuja avaliação clínica foi registrada, 31,0\% (319/1.028) eram sintomáticos (Tabela 2). Dentre os cães positivos, 55,2\% (48/87) eram assintomáticos e 44,8\% (39/ 87) apresentaram pelo menos um dos sinais clínicos sugestivos de LVC. Lesões cutâneas (24,1\%) e onicogrifose (23,0\%) foram os sinais mais freqüentes entre os soropositivos diferindo estatisticamente de ceratoconjuntivite (8,0\%) e alopecia (5,7\%). Infartamento ganglionar foi observado em apenas 1,1\% dos animais positivos e edema de membros não foi observado.

A grande maioria $(96,4 \%)$ dos quintais das residências avaliada era de terra. Em geral, encontrava-se em boas condições de sanitárias, embora apresentasse escoamento de água residual da cozinha e lavanderia. A criação, no peridomicílio,

Tabela 1. Prevalência canina de leishmaniose visceral segundo a faixa etária, município de Poxoréo-MT, 2002.

\begin{tabular}{ccccc}
\hline \multirow{2}{*}{$\begin{array}{c}\text { Faixa etária }^{\text {a }} \text { (anos) } \\
\text { Cães exami- }\end{array}$} & \multicolumn{3}{c}{ Prevalência $^{\mathrm{b}}$} \\
\cline { 3 - 5 } & nados (\%) & $\%$ & (positivo/total) & $\mathrm{IC}^{\mathrm{c}}$ \\
\hline $0-1$ & 34,5 & $6,8^{\mathrm{c}}$ & $(19 / 279)$ & $3,9-9,8$ \\
$1-3$ & 36,8 & $5,0^{\mathrm{c}}$ & $(15 / 298)$ & $2,6-7,5$ \\
$3-5$ & 15,5 & $8,8^{\mathrm{b}, \mathrm{c}}$ & $(11 / 125)$ & $3,8-13,8$ \\
$5-7$ & 8,8 & $15,5^{\mathrm{a}, \mathrm{b}}$ & $(11 / 71)$ & $7,1-23,9$ \\
$>7$ & 4,4 & $22,2^{\mathrm{a}}$ & $(8 / 36)$ & $8,6-35,8$ \\
\hline
\end{tabular}

a23 registros sem informação.

betras distintas diferem entre si $(p<0,05)$ pelo teste do Qui-quadrado. cintervalo de confiança.

Tabela 2. Sinais clínicos observados em 87 cães soropositivos para anticorpos anti-Leishmania sp., por meio da reação de imunofluorescência indireta. Poxoréo-MT, 2002.

\begin{tabular}{|c|c|c|c|c|c|}
\hline \multirow[t]{2}{*}{ Sinal clínico } & \multicolumn{2}{|c|}{ Cães examinados ${ }^{1}$} & \multicolumn{2}{|c|}{ Positivos $^{2}$} & \multirow[t]{2}{*}{$\mathrm{IC}^{3}$} \\
\hline & $\%$ & $($ n/total) & $\mathrm{n}$ & $\%$ & \\
\hline Lesões cutâneas & 18,3 & $(188 / 1028)$ & 21 & $24,1^{\mathrm{a}}$ & $15,1-33,1$ \\
\hline Onicogrifose & 17,7 & $(182 / 1028)$ & 20 & $23,0^{a}$ & $14,1-31,8$ \\
\hline Alopecia & 16,7 & $(172 / 1028)$ & 5 & $5,7^{b, c}$ & $0,9-10,6$ \\
\hline Ceratoconjuntivite & 5,6 & $(58 / 1028)$ & 7 & $8,0^{b}$ & $2,3-13,8$ \\
\hline $\begin{array}{l}\text { Infartamento } \\
\text { Ganglionar }\end{array}$ & 1,0 & $(10 / 1028)$ & 1 & $1,1^{\mathrm{c}}$ & $0,0-3,4$ \\
\hline
\end{tabular}

${ }^{1}$ cães com presença de mais de um sinal clínico

${ }^{2}$ letras distintas diferem entre si $(p<0,05)$ pelo teste do Qui-quadrado ou teste Exato de Fisher

3intervalo de confiança
Tabela 3. Características do peridomicílio segundo a positividade para leishmaniose visceral em 1.112 cães do município de Poxoréo, MT, 2002.

\begin{tabular}{|c|c|c|c|c|c|}
\hline \multirow{2}{*}{$\begin{array}{l}\text { Variáveis do } \\
\text { Peridomicílio }\end{array}$} & \multicolumn{2}{|c|}{ Registros } & \multicolumn{2}{|c|}{ Positivos } & \multirow[t]{2}{*}{$\mathrm{p}$} \\
\hline & $n$ & $\%$ & $\mathrm{n}$ & $\%$ & \\
\hline \multicolumn{6}{|c|}{ Animais co-habitando } \\
\hline Galinhas & 638 & 92,7 & 58 & 85,3 & \multirow{4}{*}{$0,0315^{c}$} \\
\hline Suínos & 32 & 4,6 & 6 & 8,8 & \\
\hline Eqüinos & 18 & 2,6 & 4 & 5,9 & \\
\hline Total & 688 & 61,9 & 68 & 78,2 & \\
\hline \multicolumn{6}{|l|}{ Coleta de lixo ${ }^{b}$} \\
\hline presente & 732 & 75,0 & 51 & 81,0 & \multirow{3}{*}{$0,2650^{d}$} \\
\hline ausente & 243 & 25,0 & 12 & 19,0 & \\
\hline Total & 975 & 100,0 & 63 & 100,0 & \\
\hline
\end{tabular}

aregistros sem informação $=424$

bregistros sem informação $=137$

cteste exato de Fisher

dteste do Qui-quadrado

de outras espécies animais que podem servir de fonte para repasto sanguíneo dos flebotomíneos foi relatada em 61,9\% dos cães avaliados (688/1.112) A análise da co-habitação de outras espécies animais com os cães soropositivos mostrou que as galinhas foram as mais freqüentes, seguidas de suínos e eqüinos, onde havia diferença estatisticamente significativa $(p=0,0315)$ entre os grupos (Tabela 3)

A coleta de lixo era realizada na maioria das residências, pelo menos uma vez por semana. No entanto, não houve associação estatisticamente significativa $(\mathrm{p}=0,2650)$ entre a coleta de lixo domiciliar e soropositividade (Tabela 3).

A renda familiar da população avaliada variou de um a 20 salários-mínimos sendo que 75,50\% (801/1061) apresentava renda familiar de um salário-mínimo, 17,3\% (184/1061) de dois salários mínimos, 32,7\% (33/1061) de três salários mínimos, 1,2\% (13/1061) de quatro salários mínimos e 2,8\% (30/ 1061) apresentavam renda maior que cinco salários mínimos.

Não se observou associação estatisticamente significativa $(p=0,1702)$ entre renda familiar e presença de cão positivo para a pesquisa de anticorpos anti-Leishmania., através da reação de imunofluorescência indireta.

\section{DISCUSSÃO}

A prevalência de leishmaniose visceral canina observada em Poxoréo (7,8\%) no presente estudo é semelhante às já relatadas para regiões endêmicas. Madeira et al. (2000) observaram 11,9\% de positividade em amostras de cães assintomáticos da região de Itaipu, RJ; Camargo-Neves et al (2001) encontraram 12,0\% de prevalência em Araçatuba, SP; França-Silva et al. (2003) observaram 9,7\% em cães da área endêmica de Montes Claros, MG. Já, outros autores têm relatado maiores prevalências como Cabrera et al. (2003) que observaram 27,7\% de positividade em cães domiciliados em Barra do Guaratiba, RJ, Paranhos-Silva et al. (1996) 23,5\% de prevalência de LVC em cães de Jequié,BA, Cortada et al. (2004) 75,3\% de positividade em cães de Anastácio, MS e Guimarães et al. (2005) que também encontraram porcenta- 
gem maior de prevalência de LVC de 21 a 25\% em cães de dois bairros de São José do Ribamar, no MA. Estes estudos tiveram influência de diversos fatores como a área avaliada, o método diagnóstico utilizado, além da amostragem utilizada uma vez que muitos estudos avaliam cães de áreas de ocorrência de LV humana constituindo-se num viés pois, não necessariamente, são representativos de todo os municípios.

Nos diferentes Setores de Poxoréo, observou-se que a prevalência variou de 2,2\% a 18,9\%. Embora estas diferenças sejam estatisticamente significativas, não foram observadas características físicas ou de densidade populacional que pudessem ser relacionadas a estes achados. França-Silva et al. (2003) observaram grande variação na prevalência de LVC nos diferentes bairros de Montes Claros, MG e sugeriram que os diferentes ecossistemas dos bairros favoreciam a manutenção dos vetores uma vez que também não observaram relação da densidade populacional canina com a prevalência. Paranhos-Silva et al. (1996) também encontraram grande variação na prevalência de diferentes estratos de Jequié, BA, porém sem relação com a densidade canina. Em ambos os casos, assim como na presente pesquisa, a avaliação da densidade de flebotomíneos não foi realizada.

Uma das dificuldades observadas na realização deste estudo foi à obtenção dos dados de maneira completa.. Muitas das fichas epidemiológicas não continham algumas informações, particularmente relativas à idade dos cães, provavelmente por esta informação depender do relato do proprietário ou da avaliação da arcada dentária, o que não foi possível em todas as coletas realizadas.

A ausência de associação estatisticamente significativa entre soro-prevalência e sexo também tem sido relatada por outros autores como Paranhos-Silva et al. (1996), Feitosa et al. (2000), Cabrera et al. (2003) e França-Silva et al. (2003) embora, Dantas-Torres et al. (2006) tenham observado maior prevalência em cães machos de Paulista, PE.

A associação da positividade com cães de idade acima de sete anos (22,2\%) também foi observada por França-Silva et al. (2003) para animais de seis a sete anos de idade (12,8\%). Moreira Jr. et al. (2003) sugeriram aumento do risco para LVC após o $1^{\circ}$ ano de vida do cão, porém não identificaram a faixa etária com maior incidência. Já Feitosa et al. (2000) relataram maior positividade em animais entre três e seis anos de idade $(29,0 \%)$, neste caso a avaliação foi realizada somente em animais positivos para LVC.

O quadro clínico da LVC varia de animais aparentemente saudáveis a aqueles em estágios finais da doença. Nesse estudo, 55,2\% dos cães soropositivos para LVC não tiveram quaisquer sinais clínicos da doença. Madeira et al. (2000) relataram positividade, embora em menor percentual (11,9\%), em cães assintomáticos da região de Itaipu, RJ. Por não apresentarem sinais clínicos, os cães assintomáticos não são facilmente associados à LVC, porém desempenham importante papel na transmissão da doença.

Dos animais soropositivos e sintomáticos, lesões cutâneas e onicogrifose foram os sinais clínicos mais freqüentemen- te observados, à semelhança do que Feitosa et al. (2000) relataram em seu estudo. Entretanto, linfoadenopatia, relatada por estes autores como o sinal clínico mais freqüente, foi observada em apenas 1,1\% dos cães soropositivos de Poxoréo. A diferença observada pode ter sido decorrente do fato de que os animais avaliados por Feitosa et al. (2000) foram oriundos do Hospital Veterinário e foram levados ao serviço devido a alguma alteração fisiológica. Os cães de Poxoréo foram aleatoriamente incluídos no presente estudo, sem necessariamente estarem com alguma alteração. Além disto, a dificuldade de se realizar exame clínico mais detalhado pode ter influenciado no resultado da presente pesquisa.

A presença de outras espécies animais no peridomicílio foi relatada em $61,9 \%$ dos casos, particularmente a presença de galinhas, criada como fonte adicional de alimentos revelou-se estatisticamente associada à soropositividade. Embora presença de galinhas não tenha apresentado associação estatisticamente significativa com a soropositividade dos cães, pode favorecer a manutenção dos flebotomíneos no peridomicílio, aumentando assim o risco de transmissão da LVC, conforme observado por Alexander et al. (2002). Moreira Jr. et al. (2003) também se referiram a maior risco de LVC quando da presença de outras espécies no peridomicílio e observaram associação significativa da LVC com a presença de suínos, associação esta não observada no presente trabalho.

A ausência de coleta de lixo domiciliar não revelou associação estatisticamente significativa com a prevalência observada em Poxoréo. Costa et al. (2005) avaliaram a influência dos serviços e saneamento básicos da cidade de Teresina,PI e observaram maior risco de infecção humana quando da ausência de rede de esgoto e coleta de lixo regulares.

A renda familiar mais freqüentemente relatada, de 1 a 2 salários-mínimos, não se revelou estatisticamente associada à soropositividade porém retrata uma população de baixa renda que pode estar vivendo em condições que favoreçam a manutenção desta zoonose.

O presente estudo revelou que a prevalência de LVC em Poxoréo foi semelhante à observada na maioria das áreas endêmicas, que a doença esteve presente em toda a área urbana e que esta ocorrência não esteve associada às condições econômicas dos proprietários. Por outro lado, fatores como idade dos cães superior a 7 anos e a presença de outra espécie animal co-habitando com os cães podem ser fatores de risco para a leishmaniose visceral canina e podem ser considerados quando da aplicação das medidas de controle.

Agradecimentos:- Ao Laboratório Central do Estado do Mato Grosso pela realização dos exames de RIFI; ao Coordenador e Agentes de Saúde da FUNASA de Poxoréo, MT pelo apoio logístico e na coleta das amostras.

\section{REFERÊNCIAS BIBLIOGRÁFICAS}

ALENCAR, J.E. Expansão do calazar no Brasil. Ceará Medicina, v. 5, n.1-2, p.86-102, 1983. 
ALEXANDER, B.; CARVALHO, R.L.; McCALLUM, H.; PEREIRA, M.H. Role of domestic chicken (Gallus gallus) in the epidemiology of urban visceral leishmaniasis in Brazil. Emerging Infectious Diseases, v. 8, n.12, p.1480-1485, 2002.

BADARO, R.; JONES, T.C.; LORENÇO, R.; CERF, B.J. SAMPAIO, D. CARVALHO, E.M.; ROCHA, H.; TEIXEIRA, R.; JOHNSON, W.R. A prospective study of visceral leishmaniasis in an endemic area of Brazil. Journal of Infectious Disease, v.54, n.4, p. 639-649, 1986.

BRASIL, Instituto Brasileiro de Geografia e Estatística, Ministério do Planejamento, Orçamento e Gestão, Brasil. Resultados da Amostra do Censo Demográfico, 2000. Disponível em:<http:// www.ibge.gov.br>. Acesso em: 22 mar. 2006.

CABRERA, M.A.; PAULA, A.A.; CAMACHO, L.A.; MARZOCHI, M.C.; XAVIER, S.C.; SILVA, A.V.; JANSEN, A.M. Canine visceral leishmaniais in Barra de Guaratiba, Rio de Janeiro, Brazil: assessment of risk factors. Revista do Instituto de Medicina Tropical, v.25, n.2, p.79-83, 2003.

CAMARGO M.E., REBONATO C. Cross reactivity in fluorescence tests for Trypanosoma and Leishmania antibodies. American Journal of Tropical Medicine and Hygiene, v.18, n.4, p.500-505, 1969.

CAMARGO-NEVES, V.L.F.; KATZ, G.; RODAS, L.A.C.; POLETTO, D.W.; LAGE, L.C.; SPÍNOLA, R.M.F.; CRUZ, O.G. Utilização de ferramentas de análise espacial na vigilância epidemiológica de leismaniose visceral americanaAraçatuba-São Paulo, Brasil, 1998-199. Cadernos de Saúde Pública, v.17, n.5, p.1263-1267, 2001.

CIFUENTES, E.E. Program for the elimination of urban rabies in Latin America. Review Infectious Disease, v.10, supl.4, p.S689-S692, 1988.

CORTADA, V.M.C.L.; DOVAL, M.E.C.; SOUZA LIMA, M.A.A.; OSHIRO, E.T.; NENESES, C.R.V.; ABREU-SILVA, A.L.; CUPOLILO, E.; SOUZA, C.S.F.; CARDOSO, F.O.; ZAVERUCHA DO VALLE, T.; BRAZIL, R.P.; CALABRESE, K.S.; GONÇALVES DA COSTA, S.C. Canine visceral leishmaniasis in Anastácio, Mato Grosso do Sul State, Brazil. Veterinary Research Communications, v.28, n.5, p.365-374, 2004.

COSTA C.H.; WERNECK, G.L.; RODRÍGUEZ, L. Jr.; SANTOS, M.V.; ARAUJO, I.B.; MOURA, L.S., MOREIRA, S.; GOMES, R.B.; LIMA, S.S. Household structures and urban services: neglected targets in the control of visceral leishmaniasis. Annals of Tropical Medicine and Parasitology, v.99, n.3, p.229-236, 2005.

DANTAS-TORRES, F.; BRITO, M.E.F.; BRANDÃO-FILHO. Seroepidemiological survey on canine leishmaniasis among dogs from an urban area of Brazil. Veterinary Parasitology, v.140, n.1-2, p.54-60, 2006.

FEITOSA, M.M.; IKEDA, F.A.; LUVIZOTO, M.C.; PERRI, S.H.; Aspectos clínicos de cães com leishmaniose visceral no município de Araçatuba- SP, Brasil. Clínica Veterinária, v.5, n. 28, p.36-44, 2000.

FRANÇA-SILVA, J.C.; COSTA, R.T.; SIQUEIRA, A. M.; MACHADO-COELHO, G.L.L.; COSTA, C.A.; MAYRINK, W.; VIEIRA, E.P.; COSTA, J.S.;GENNARO, O., NASCIMENTO, E. Epidemiology of canine visceral leishmaniasis in the endemic area of Montes Claros municipality, Minas Gerais State, Brazil. Veterinary Parasitology, v.111, n.2-3, p.161-173, 2003.

GONTIJO, C.M.F.; MELO, M.N. Leishmaniose visceral no Brasil: quadro atual, desafios e perspectivas. Revista Brasileira de Epidemiologia, v.7, n.3, p.338-349, 2004.

GRAMICCIA, M.; GRADONI, L. The current status of zoonotic leishmaniasis and approaches to disease control. International Journal for Parasitology, v.35, n.11-12, p.1169-1180, 2005.

GUIMARÃES, K.S.; BATISTA, Z.S.; DIAS, E.L.; GUERRA, R.M.S.N.C.; COSTA, A.D.C.; OLIVEIRA, A.S.; CALABRESE, K.S.; CARDOSO, F.O; SOUZA, C.S.F.; ZAVERUCHA DO VALE, T.; GONÇALVES DA COSTA, S.C.; ABREU-SILVA, A.L. Canine visceral leishmaniasis in São José de Ribamar, Maranhão State, Brazil. Veterinary Parasitology, v.131, n. 3-4, p.305-309, 2005.

LWANGA, S.K.; LEMESHOW, S. Sample size determination in health studies. Genebra: WHO, 1991. 80 p.

MADEIRA, M.F.; SERRA, C. M.; UCHOA, C.M.; DUARTE, R.; CRUZ, D.A.; PERDOMO, C.C. Leishmaniose canina: avaliação sorológica de 310 cães na região de Itaipu, Rio de Janeiro. Cadernos de Saúde Pública, v.16, n.2, p.568, 2000.

MONTEIRO, P.S.; LACERDA, M.M.; ARIAS, J.R. Controle da leishmaniose visceral no Brasil. Revista da Sociedade Brasileira de Medicina Tropical, v.27, supl. 3, p.64-72, 1994. MOREIRA Jr., E.D.; SOUZA, V.M.M.; SREENIVASAN, M.; LOPES, N.L.; BARRETO, R.B.; CARVALHO, L.P. Peridomestic risk factors for canine leishmaniasis in urban dwellings: new findings from a prospective study in Brazil. American Journal of Tropical Medicine and Hygiene, v.69, n.4, p.393-397, 2003.

MOREIRA Jr.; SOUZA, V.M.M.; SREENIVASAN, M.; NASCIMENTO, E.G.; CARVALHO, L.P. Assesment of an optimized dog-culling program in the dynamics of canine Leishmania transmission. Veterinary Parasitology, v.122, n.4, p.245-252, 2004.

PARANHOS-SILVA, M.; FREITAS, L.A.R.; SANTOS, W.C.; GRIMALDI Jr, G.; CARVALHO, L.P.; OLIVEIRA-DOSSANTOS, A.J. A cross-sectional serodiagnostic survey of canine leishmaniasis due to Leishmania chagasi. American Journal of Tropical Medicine Hygiene, v.55, n.1, p.39-44, 1996.

SAS Institute Inc., Version 8, Cary: SAS Institute Inc., 1999.

Recebido em 10 de maio de 2006.

Aceito para publicação em 12 de agosto de 2008. 